\title{
Multilocus BOLD Dataset for Nearctic Polycentropodidae (Trichoptera): Metadata
}

Alexander Orfinger (1D, Andrew Rasmussen \& Raymond Hix
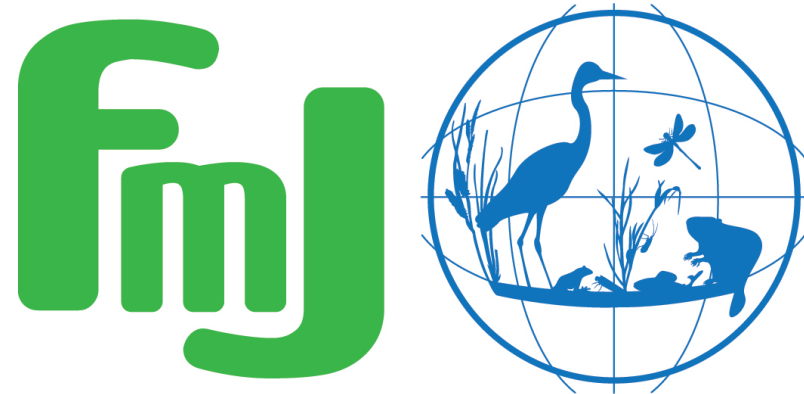

Freshwater Metadata Journal 



\title{
Multilocus BOLD Dataset for Nearctic Polycentropodidae (Trichoptera): Metadata
}

\author{
Alexander Orfinger ${ }^{1,2}$ (iD , Andrew Rasmussen ${ }^{1}$ \& Raymond Hix ${ }^{1}$ \\ 1 Florida A\&M University, College of Agriculture and Food Sciences, Tallahassee, United States; corresponding author: \\ aborfinger@gmail.com \\ 2 University of Florida, Department of Entomology and Nematology, Gainesville, United States
}

Please cite this paper as follows: Orfinger, A.B., Rasmussen, A.K. \& Hix, R.L., 2021. Multilocus BOLD Dataset for Nearctic Polycentropodidae (Trichoptera): Metadata. Freshwater Metadata Journal 51: 1-6. https://doi.org/10.15504/fmj.2021.51

Received: 2021-02-06 / Published: 2021-02-16

\section{Keywords}

Annulipalpia, biodiversity, caddisflies, distribution, DNA barcoding, phylogeny, taxonomy

\section{Short description of the dataset/summary}

The BOLD dataset "DS-POLYCSS Nearctic Polycentropodidae (Trichoptera)" contains publicly accessible molecular data and associated voucher collection data. Data include two loci (nuclear 28s rRNA D2 and mitochondrial cytochrome oxidase $\mathrm{C}(\mathrm{COI})$ ), specimen images, collection data, and additional specimen data. Data pertain to hundreds of treated specimens and dozens of treated species, of different life stages and both sexes, of the Trichoptera family Polycentropodidae in the Nearctic, with an emphasis on Polycentropus sensu lato. Data will continued to be added during continued systematic studies of the family by the authors.

\section{General information}

dataset entry ID:

name of the dataset:

full name of the dataset:

dataset short name:

type of dataset:

data type:

\section{FWM_31}

Multilocus BOLD Dataset for Nearctic Polycentropodidae (Trichoptera) DS-POLYCSS Nearctic Polycentropodidae others descriptive data

science keywords according to GCMD:

topic:

Biosphere, Biological Classification

ISO topic category according to ISO 19115:

INSPIRE keywords according to GEMET:

Biota, Inland Waters

Species distribution 
own science keywords:

related project:

funding: aquatic insects, Annulipalpia, biodiversity, DNA barcoding, phylogeny, taxonomy, Trichoptera

BOLD ORFIN - Nearctic Polycentropus sensu lato (Trichoptera:

Polycentropodidae)

This work was supported by the McIntire-Stennis Program from the USDA

National Institute of Food and Agriculture and by the USDA National Institute of Food and Agriculture, 1890 Institution Capacity Building Grant Project 1021805.

\section{Technical and administrative specifications}

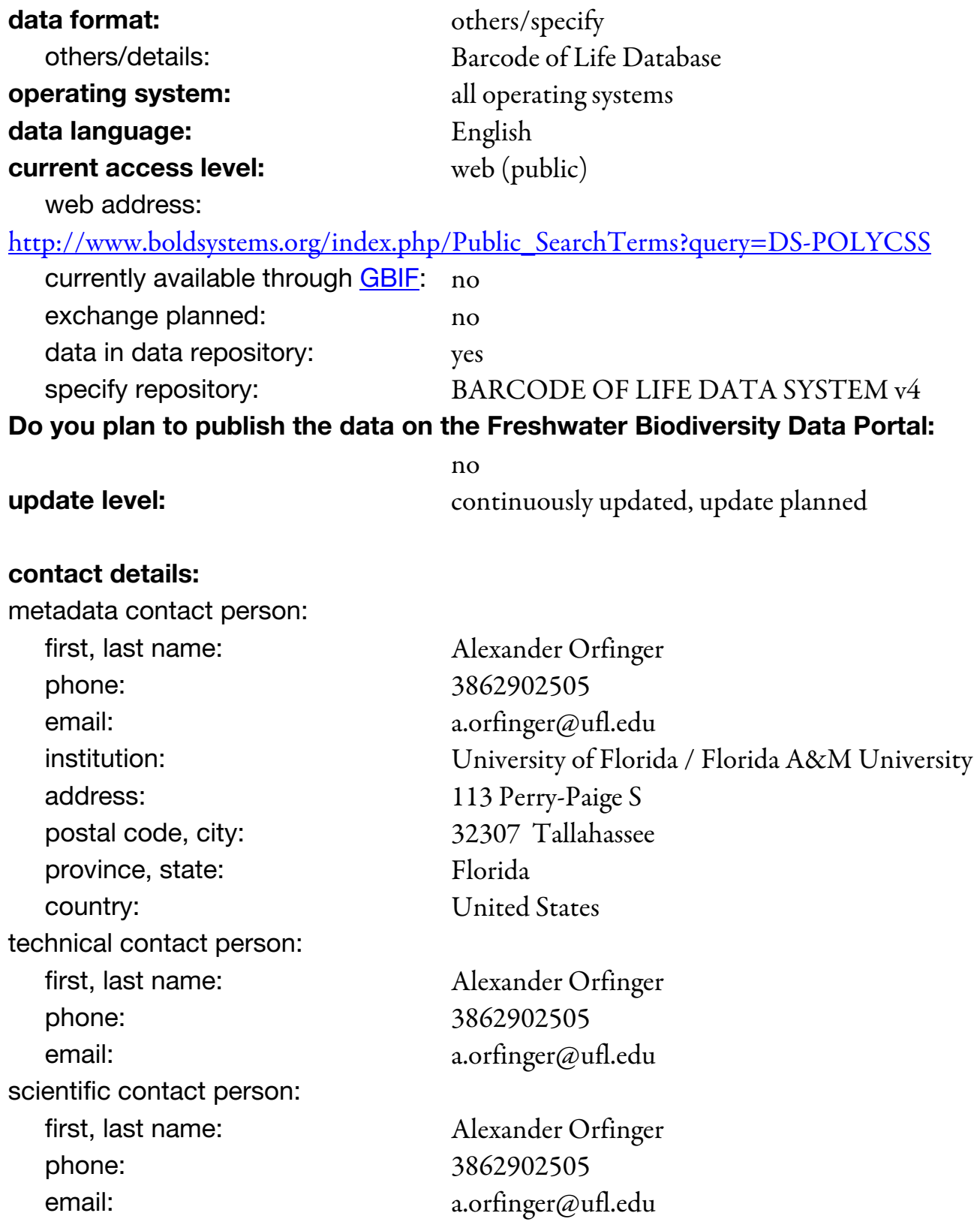

\section{contact details:}

metadata contact person:

first, last name:

phone:

email:

institution:

address:

postal code, city:

province, state:

country:

technical contact person:

first, last name:

phone:

email:

scientific contact person:

first, last name:

phone:

email:

\author{
Alexander Orfinger \\ 3862902505 \\ a.orfinger@ufl.edu \\ University of Florida / Florida A\&M University \\ 113 Perry-Paige $S$ \\ 32307 Tallahassee \\ Florida \\ United States \\ Alexander Orfinger \\ 3862902505 \\ a.orfinger@ufl.edu \\ Alexander Orfinger \\ 3862902505 \\ a.orfinger@ufl.edu
}




\section{Intellectual property rights and citation}

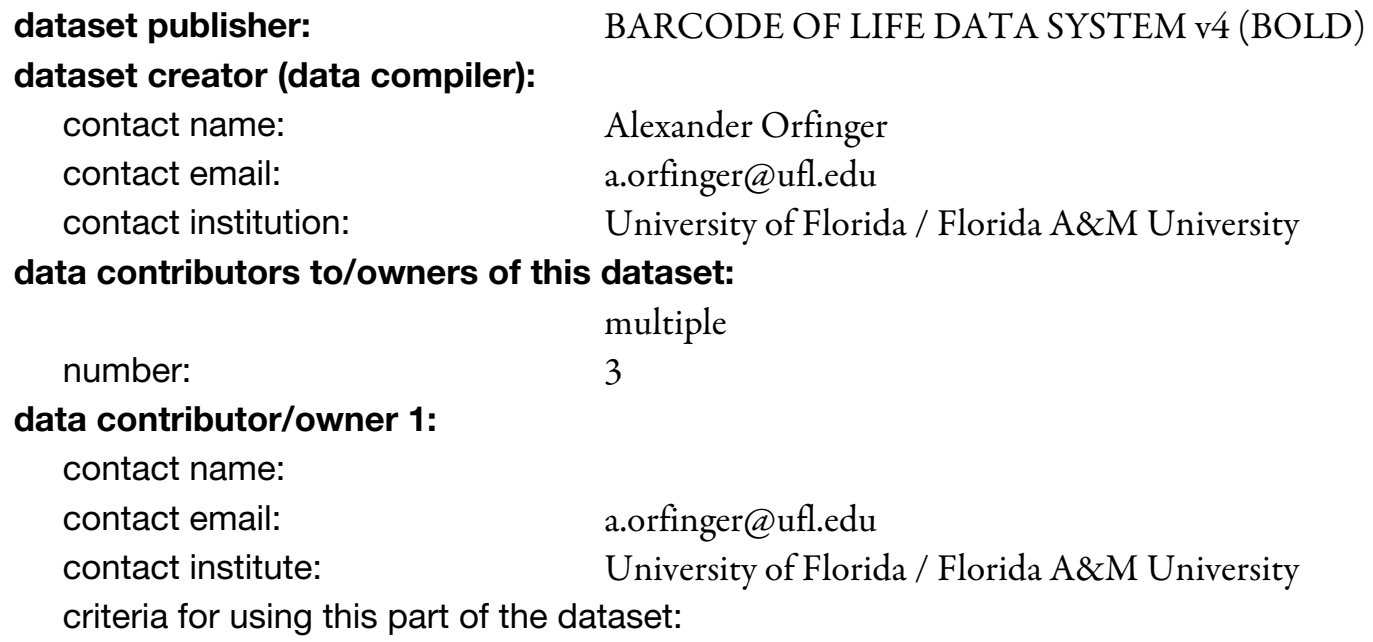

The dataset is publicly available (data portal, data archive) and can be used without restrictions, but dataset creator/data contributors must be informed prior to publication. Data must be acknowledged and cited correctly.

data contributor/owner 2:

contact name:

contact email:

andrew.rasmussen@famu.edu

contact institute:

Florida A\&M University

criteria for using this part of the dataset:

The dataset is publicly available (data portal, data archive) and can be used without restrictions, but dataset creator/data contributors must be informed prior to publication. Data must be acknowledged and cited correctly.

data contributor/owner 3:

contact name:

contact email:

raymond.hix@famu.edu

contact institute:

Florida A\&M University

criteria for using this part of the dataset:

The dataset is publicly available (data portal, data archive) and can be used without restrictions, but dataset creator/data contributors must be informed

citation of this dataset: prior to publication. Data must be acknowledged and cited correctly.

author(s):

Orfinger, A.B., Rasmussen, A.K. \& Hix, R.L.

title and journal (name, number, pages):

Dataset - DS-POLYCSS Nearctic Polycentropodidae (Trichoptera),

BARCODE OF LIFE DATA SYSTEM v4, 2021, accessible at http://www.boldsystems.org/index.php/Public_SearchTerms?query=DS-POL YCSS

year: 2021

doi: $\quad$ https://doi.org/10.5883/DS-POLYCSS

citation of the metadata:

author(s): $\quad$ Orfinger, A.B., Rasmussen, A.K. \& Hix, R.L.

title and journal (name, number, pages):

Multilocus BOLD Dataset for Nearctic Polycentropodidae (Trichoptera):

Metadata. Freshwater Metadata Journal 51: 1-6

year:

2021

doi: $\quad$ https://doi.org/10.15504/fmj.2021.51 


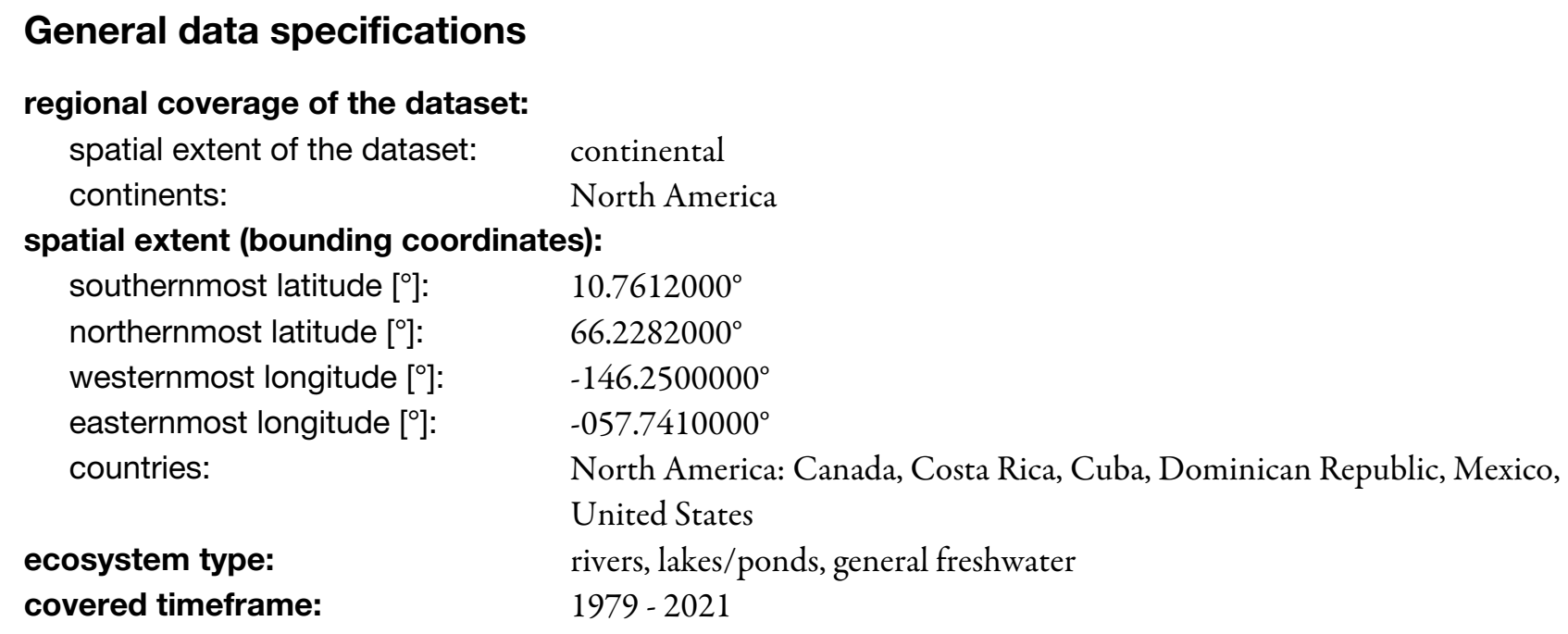

\section{Site specifications}

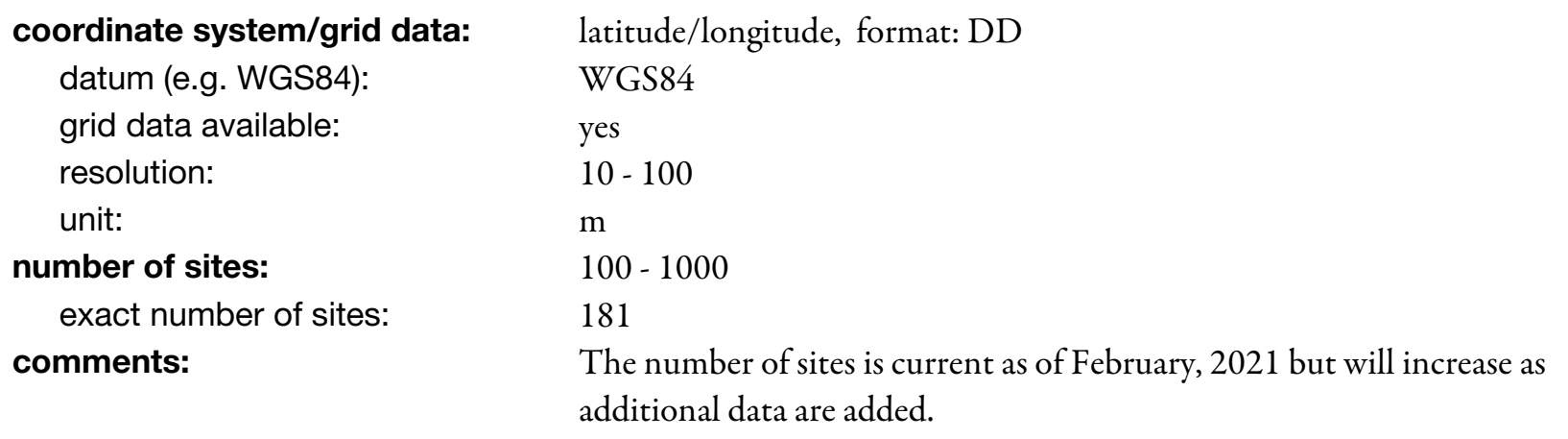

\section{Climate and environmental data}

\section{climate related data: \\ environmental data:}

available parameters per site: no climate data available

no environmental data per catchment available

information on riparian vegetation (incl. information on modification) data source: collector(s)

information on embankment (incl. information on modification) data source: collector(s)

information on channel form (incl. information on modification) data source: collector(s)

information on water uses (e.g., irrigation, fish ponds) data source: collector(s)

distance to the next main village/town upstream data source: collector(s)

stream order (according to Strahler) data source: collector(s)

altitude data source: collector(s)

information on instream habitat (incl. information on modification) data source: collector(s) 
physico-chemical data:

stressors influencing the sites: no physico-chemical data available

no stressor data available

\title{
Biological data
}

\author{
biological data origin: from sampling, \\ various projects \\ organism group addressed: macro-invertebrates (Trichoptera)
}

\section{Sample specifications/sample resolution}

\section{macro-invertebrates: \\ sample information:}

covered timeframe:

$1979-2021$

historical data:

yes

palaeo data:

no

season:

spring, summer, autumn, winter

temporal resolution/frequency of sampling:

comments:

infrequent sampling frequency

Data collection and input are ongoing.

\section{taxonomic resolution:}

level:

order, family, sub-family, genus, species

percentage of species level data:

comments:

85

Specimens are identified to species when possible.

\section{taxonomic coding:}

taxalist according to:

reference(s):

Trichoptera Nearctica and Trichoptera World Checklist

- Rasmussen, A.K. \& Morse, J.C., 2020. Distributional Checklist of Nearctic Trichoptera (Fall 2020 Revision). Unpublished, Florida A\&M University,

Tallahassee. 517 pp. [Available at http://www.Trichoptera.org]

- Morse, J.C. (ed.), 2021. Trichoptera World Checklist.

http://entweb.clemson.edu/database/trichopt/index.htm

\section{sample specifications:}

type:

qualitative, presence/absence

replicate samples:

yes

specification of method(s) used for sampling and sorting:

Sampling methods varied according to life stage and collector. Collection method data are available within the individual specimens' "Specimen Page" when known. Collection methods included UV light trapping, sweep netting, malaise trapping, and emergence trapping for adults, and hand picking and standard benthic collecting methods for immature stages.

\section{Other specifications}

\section{GIS layers, shape files related to the dataset:}

no data available 


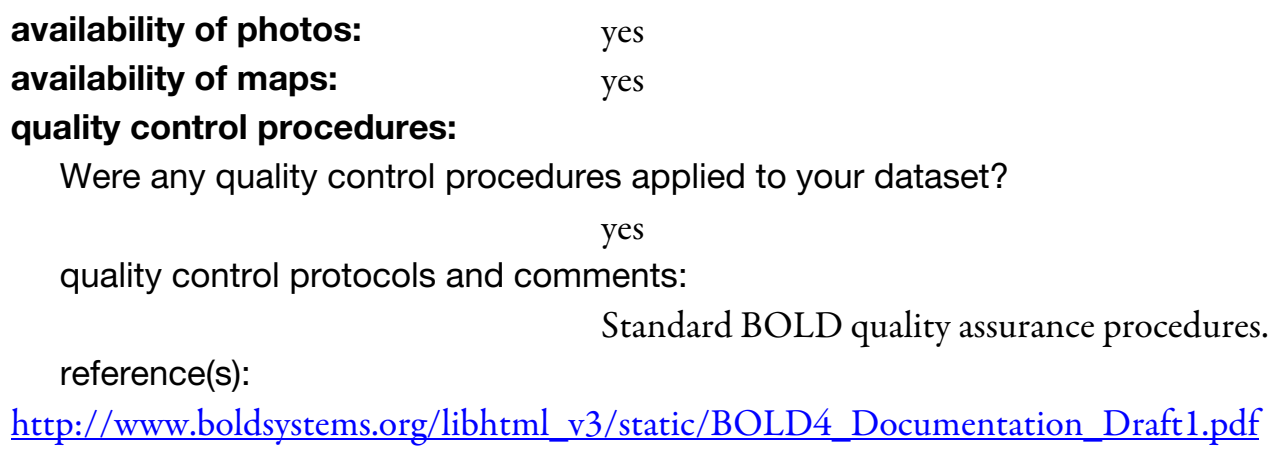

\section{Acknowledgements}

This work was supported by the McIntire-Stennis Program from the USDA National Institute of Food and Agriculture and by the USDA National Institute of Food and Agriculture, 1890 Institution Capacity Building Grant Project 1021805. Numerous museums and researchers provided specimens and data for production of this data set, for which the authors are very grateful. The authors also appreciate the helpful review of Dr. Astrid Schmidt-Kloiber (BOKU, Vienna).

\section{References}

Morse, J.C. (ed.), 2021. Trichoptera World Checklist. http://entweb.clemson.edu/database/trichopt/index.htm

Rasmussen, A.K. \& Morse, J.C., 2020. Distributional Checklist of Nearctic Trichoptera (Fall 2020 Revision). Unpublished, Florida A\&M University, Tallahassee. 517 pp. [Available at http://www.Trichoptera.org]

Ratnasingham, S. \& Hebert, P.D., 2007. BOLD: The Barcode of Life Data System (http://www. barcodinglife. org). Molecular Ecology Notes, 7(3), 355-364. https://doi.org/10.1111/j.1471-8286.2006.01678.x 